\title{
PRUEBAS TOXICOLÓGICAS PREOCUPACIONALES PARA EL CONSUMO DE DROGAS EN EMPRESAS CATALOGADAS COMO DE ALTO RIESGO
}

Recibido: octubre del 2018

Aceptado: abril del 2019

Guillermo A. Castaño Pérez ${ }^{1}$, Carolina Salas Zapata ${ }^{2}$

\section{Resumen}

Introducción: El consumo de alcohol y de drogas en ambientes laborales constituye un riesgo para el mismo trabajador, para terceros y compromete la productividad. La OIT recomienda la evaluación médica previa de la salud de los trabajadores. El objetivo de este trabajo fue estimar la prevalencia de consumo de marihuana y cocaína en muestras de orina para aspirantes a empleos de alto riesgo y analizar algunos factores asociados. Métodos: Se tomaron 2.197 muestras de orina para detectar consumo de marihuana y cocaína. La fuente fue secundaria. El análisis toxicológico se realizó con la técnica de inmunoensayo cromatográfico de flujo lateral. Se calcularon medidas de frecuencia absoluta y relativa, y se aplicó la prueba de chi cuadrado de Pearson para el análisis de factores asociados. Resultados: La prevalencia de marihuana fue de 7,3\% y de cocaína de 3,6\%. Hubo asociación estadística entre el resultado positivo de marihuana y sexo, edad, escolaridad y tipo de empresa. Para cocaína se encontró asociación con las mismas variables, excepto en edad. Conclusiones: El uso de pruebas de drogas en orina como exámenes médicos preocupacionales es el primer paso para la prevención del consumo de drogas en ambientes laborales de alto riesgo. Hay evidencia acerca de que los aspirantes a un trabajo que consumen drogas tienen más probabilidad de asumir riesgos en el ambiente laboral.

Palabras clave: cannabis, cocaína, drogas, preocupacionales, pruebas

toxicológicas, salud laboral.

${ }^{1}$ Médico, magíster en Drogodependencias, PhD en Piscología de la Salud. Grupo de investigación en Salud Mental, Facultad de Medicina, Universidad CES. Correo: gacastano@ ces.edu.co

2 Gerente de Sistemas de Información en Salud, magíster en Epidemiología. Grupo de Investigación en Salud Mental, Facultad de Medicina, Universidad CES. Correo: csalas@ces. edu.co 


\title{
PRE-OCCUPATIONAL TOXICOLOGICAL TESTING FOR DRUG CONSUMPTION IN COMPANIES CLASSIFIED AS HIGH-RISK
}

\author{
Guillermo A. Castaño Pérez' ${ }^{1}$ Carolina Salas Zapata²
}

\section{Abstract}

Introduction: Consumption of alcohol and drugs in work environments suppose a risk for the employees, for third parties and for the productivity. World's ILO recommends previous medic evaluation of employees' health. The objective of this work was to estimate the prevalence of marihuana consumption and cocaine in urine samples for candidates to high-risk jobs and to analyze the associated factors. Method: We took 2.197 urine samples to detect any consumption of marihuana or cocaine. The source was secondary. Toxicological analysis was made with a technique of chromatographic immune-essay of lateral flux. Measures for absolute and relative frequency were calculated, as well as chi-squared tests for the analysis of the associated factors. Results: Prevalence of marihuana was of $7.3 \%$ and cocaine of $3.6 \%$. There was a statistical association between the positive result of marihuana and the sex, age, education and type of company. For cocaine, the same variables were associated, except the age. Conclusions: Using drug tests for urine as medical pre-occupational examinations is the first step to prevent drug consumption in high-risk work environments. There is evidence that candidates for a job who consume drugs have bigger chances to assume risks in the work environment. 


\title{
EXAMES TOXICOLÓGICOS PRÉ-OCUPACIONAIS PARA O CONSUMO DE DROGAS EM EMPRESAS CATALOGADAS COMO DE ALTO RISCO
}

\author{
Guillermo A. Castaño Pérez ${ }^{1}$, Carolina Salas Zapata ${ }^{2}$
}

\section{Resumo}

Introdução: o consumo de álcool e de drogas em ambientes corporativos constitui um risco para o trabalhador e para terceiros, além de comprometer a produtividade. A Organização Internacional do Trabalho recomenda a avaliação médica prévia da saúde dos trabalhadores. O objetivo deste trabalho foi estimar a prevalência de consumo de maconha e cocaína em amostras de urina para candidatos a empregos de alto risco e analisar alguns fatores associados. Métodos: foram tomadas 2.197 amostras de urina para detectar o consumo de maconha e cocaína. A fonte foi secundária. $\mathrm{O}$ exame toxicológico foi realizado com a técnica de imuno-ensaio cromatográfico de fluxo lateral. Foram calculadas medidas de frequência absoluta e relativa, e foi aplicado o teste de Qui-Quadrado de Pearson para a análise de fatores associados. Resultados: a prevalência da maconha foi de 7,3\% e da cocaína de 3,6\%. Houve associação estatística entre o resultado positivo de maconha e sexo, idade, escolaridade e tipo de empresa. Para a cocaína, foi verificada associação com as mesmas variáveis, exceto em idade. Conclusões: o uso de testes de drogas em urina como exames médicos pré-ocupacionais é o primeiro passo para prevenir o consumo de drogas em ambientes de trabalho de alto risco. Há evidência acerca de que os candidatos a um trabalho que consomem drogas têm mais possibilidade de assumir riscos nesse contexto. 


\section{Introducción}

Las condiciones de trabajo son una parte importante en la vida y pueden tener una decisiva influencia en los niveles de salud y bienestar, dentro y fuera del ámbito laboral. La prevención de riesgos laborales es un deber de las empresas y un derecho de los trabajadores, los cuales también deben velar por la protección de su propia salud y la de sus compañeros. La legislación en general considera un objetivo básico de las políticas laborales la protección de la salud de los trabajadores, mediante la prevención de riesgos directamente derivados del trabajo y de los que pueden afectar el rendimiento laboral.

Según las recomendaciones de la Organización Mundial de Salud (OMS) (1) es preciso adoptar medidas para reducir al mínimo las diferencias que existen entre los diversos grupos de trabajadores, en lo que respecta a los niveles de riesgo y el estado de salud.

La gravedad de los problemas por consumo de alcohol u otras drogas en el medio laboral ha sido reconocida desde hace tiempo por la Organización Internacional del Trabajo (OIT), la cual considera que el consumo de alcohol y de otras drogas es un problema que concierne a un número elevado de trabajadores (2). Se señala que los efectos nocivos del consumo de alcohol y de drogas en el lugar de trabajo y en el rendimiento laboral son frecuentes a todos los niveles. La seguridad profesional y la productividad pueden verse afectadas de forma adversa por empleados bajo el influjo del alcohol o de las drogas (3).
La OIT indica también cómo el consumo de estas sustancias, por parte de los trabajadores, produce un fuerte impacto que se extiende a la familia y a los compañeros de trabajo que son afectados en términos de pérdida de ingresos, estrés y baja moral (4). Por tanto, el consumo de alcohol y de otras drogas es un problema que no solo afecta al consumidor, sino también al ambiente laboral y a la eficacia de una empresa. Entre las evaluaciones que recomienda la OIT, está incluida la evaluación médica preocupacional de la salud de los trabajadores antes de que se les asignen tareas específicas que puedan entrañar un peligro para su salud o para la de los demás.

Con relación a la evaluación médica preocupacional, esta tiene por objetivo determinar el estado de salud al momento del ingreso y su aptitud hacia el puesto de trabajo. Para trabajos de alto riesgo, definidos estos como las tareas que por su naturaleza o lugar donde se realiza, implican la exposición o la intensidad mayor a las normalmente presentes en la actividad rutinaria, las cuales pueden causar accidentes laborales severos y en muchas ocasiones, mortales (5), es frecuente que en los exámenes complementarios preocupacionales se incluyan pruebas tamiz de drogas en orina (6).

Con respecto a los trabajos de riesgo, el Decreto 2090 de 2003 (7), en su artículo 2 señala taxativamente las siguientes actividades: 1. Trabajos en minería que impliquen prestar el servicio en socavones o en subterráneos; 2 . Trabajos que impliquen la exposición a altas temperaturas, por encima de los valores límites permisibles; 3. Trabajos con exposición 
a radiaciones ionizantes; 4. Trabajos con exposición a sustancias comprobadamente cancerígenas; 5 . Pilotos de aeronaves, técnicos aeronáuticos con funciones de controladores de tránsito aéreo; 6. Cuerpos de bomberos con la actividad relacionada con la función específica de actuar en operaciones de extinción de incendios; 7. Personal del Instituto Nacional Penitenciario y Carcelario, INPEC, dedicado a la custodia y vigilancia de los internos en los centros de reclusión carcelaria.

De otro lado y complementario a lo anterior, el Decreto 1108 de 1994 (8), por el cual se sistematizan, coordinan y reglamentan algunas disposiciones en relación con el porte y consumo de estupefacientes y sustancias psicotrópicas, en su capítulo X, artículo 41, indica:

Aquellas personas cuya actividad implica un riesgo para los demás o que son de responsabilidad respecto de terceros, no podrán usar o consumir estupefacientes o sustancias psicotrópicas durante el desarrollo de su actividad, de conformidad con las normas previstas en los reglamentos y códigos que regulan el ejercicio de la respectiva profesión u oficio.

Se señalan como actividades de riesgo: conductores de cualquier tipo de vehículos; pilotos de naves y aeronaves; alumnos de pilotaje, instructores de vuelo; maquinistas y operarios; médicos, odontólogos y demás profesionales de la salud; quienes manipulan o tienen bajo su cuidado materiales o sustancias combustibles o inflamables; explosivos, sustancias tóxicas, venenosas, corrosivas o radiactivas; quienes portan o trans- portan armas; operadores y controladores aéreos y en general personal técnico de mantenimiento y apoyo de aeronaves en tierra.

Amparados en estas normativas, un grupo de empresas en Medellín que ostentan la certificación BASC (Business Alliance for Secure Commerce), alianza empresarial internacional que promueve un comercio seguro en cooperación con gobiernos y organismos internacionales, en un esfuerzo por mantener las compañías libres de cualquier actividad ilícita, entre ellas el consumo de drogas entre sus trabajadores y facilitar los procesos aduaneros de las misma, realizan en los exámenes complementarios preocupacionales pruebas toxicológicas de drogas en orina, para descartar aquellos aspirantes consumidores de drogas, con el fin de minimizar los riesgos de tráfico de drogas en sus mercancías, mejorar la productividad y además disminuir la accidentalidad laboral, con perjuicio para el mismo aspirante y para terceros.

El objetivo del estudio fue estimar la prevalencia del consumo de marihuana y de cocaína a través de pruebas toxicológicas en muestras de orina en un grupo de aspirantes a empleos en empresas con oficios clasificados como de alto riesgo y analizar algunos de sus factores asociados.

\section{Materiales y métodos}

Durante el período 2010-2017, se tomaron 2.197 muestras de orina de personas aspirantes a vacantes para empleos de alto riesgo de empresas del sector transporte, manufactura, vigilancia privada, 
exportadoras de flores y construcción. La prueba fue solicitada dentro de los exámenes complementarios de la valoración preocupacional. Todos los aspirantes fueron informados del examen al que iban a ser sometidos, además se les explicó sobre la política de la empresa con respeto al consumo de drogas y firmaron el consentimiento informado donde aceptaban voluntariamente realizarse la prueba y autorizaba a la institución que las realizaba a reportar los resultados a la empresa. Se dio cumplimiento a lo establecido en la Resolución 8430 de 1993, respecto a los requisitos para investigaciones en Colombia y la Declaración de Helsinki para el estudio primario.

El presente análisis se realizó con fuente secundaria de información, correspondiente a la base de datos suministrada por la entidad que realizó la toma de muestras y las pruebas tamiz, garantizando la confidencialidad de los datos allí incluidos, los cuales fueron suministrados en una base de datos general, sin identificar a los sujetos. Las muestras de orina se analizaron para marihuana y cocaína por ser estas las sustancias ilegales más consumidas en la población general (9).

El análisis toxicológico se realizó con la técnica de inmunoensayo cromatográfico de flujo lateral, diseñado para detectar la presencia de drogas en orina humana en concentraciones límite (cutoff), establecido para muestras positivas a través del Samhsa (Substance Abuse and Mental Health Services Administration) y el Ewdts (European Workplace Drug Testing Society) (10). La prueba de detección de drogas THC/COC fue en Casete y se usó la marca RightSign ${ }^{\circledR}$.

El análisis estadístico se llevó a cabo utilizando el software SPSS versión 23, licencia de la Universidad CES. Para la caracterización de la población se utilizaron medidas de frecuencia absoluta y relativa. Para las variables cuantitativas se calcularon medidas de resumen. Las asociaciones entre el resultado de las pruebas toxicológicas y las características de los empleados se analizaron en tablas cruzadas bivariadas para cada sustancia, por medio de la prueba estadística de Chi-cuadrado de Pearson, al tener como referencia un valor $\mathrm{p}<0,05$.

\section{Resultados}

\section{Características sociodemográficas de los sujetos y las empresas}

La edad promedio de los participantes fue de 28,9 años $(\mathrm{DE}=8,1)$, siendo mayor la edad promedio de las personas con resultado positivo para cocaína que las que obtuvieron dicho resultado para marihuana (27,9 y 26,0 años respectivamente). La mayoría de los participantes eran hombres $(89,1 \%)$ y el nivel de escolaridad que predominó fue bachillerato. En cuanto al tipo de empresas, se observó una mayor participación por parte del sector manufacturero y del de transporte (56,2\% y $33,0 \%)$ (Tabla 1$)$. 
Tabla 1. Características de los sujetos del estudio y las empresas

\begin{tabular}{llc|c|}
\hline \multicolumn{2}{c|}{ Características } & Frecuencia & $\%$ \\
\hline \multirow{2}{*}{ Sexo } & Femenino & 240 & $10,9 \%$ \\
& Masculino & 1.957 & $89,1 \%$ \\
Grupos de edad & $14-25$ & 912 & $41,5 \%$ \\
& $26-35$ & 866 & $39,4 \%$ \\
& $36 y$ más & 419 & $19,1 \%$ \\
Escolaridad & Bachillerato & 1.005 & $45,7 \%$ \\
& Primaria & 78 & $3,6 \%$ \\
& Profesional & 159 & $7,2 \%$ \\
& Sin datos & 609 & $27,7 \%$ \\
& Técnico & 212 & $9,6 \%$ \\
& Tecnólogo & 134 & $6,1 \%$ \\
& Transporte & 724 & $33,0 \%$ \\
& Manufactura & 1.235 & $56,2 \%$ \\
& Vig. privada & 122 & $5,6 \%$ \\
& Agrícola & 28 & $1,3 \%$ \\
& Construcción & 88 & $4,0 \%$ \\
\hline
\end{tabular}

Fuente: elaboración propia.

Resultados toxicológicos dela población Ambas sustancias predominaron en las personas de sexo masculino (figura $1 \mathrm{y}$

Se encontró una prevalencia de mari- tabla 2).

huana de 7,3\% y de 3,6\% para cocaína.

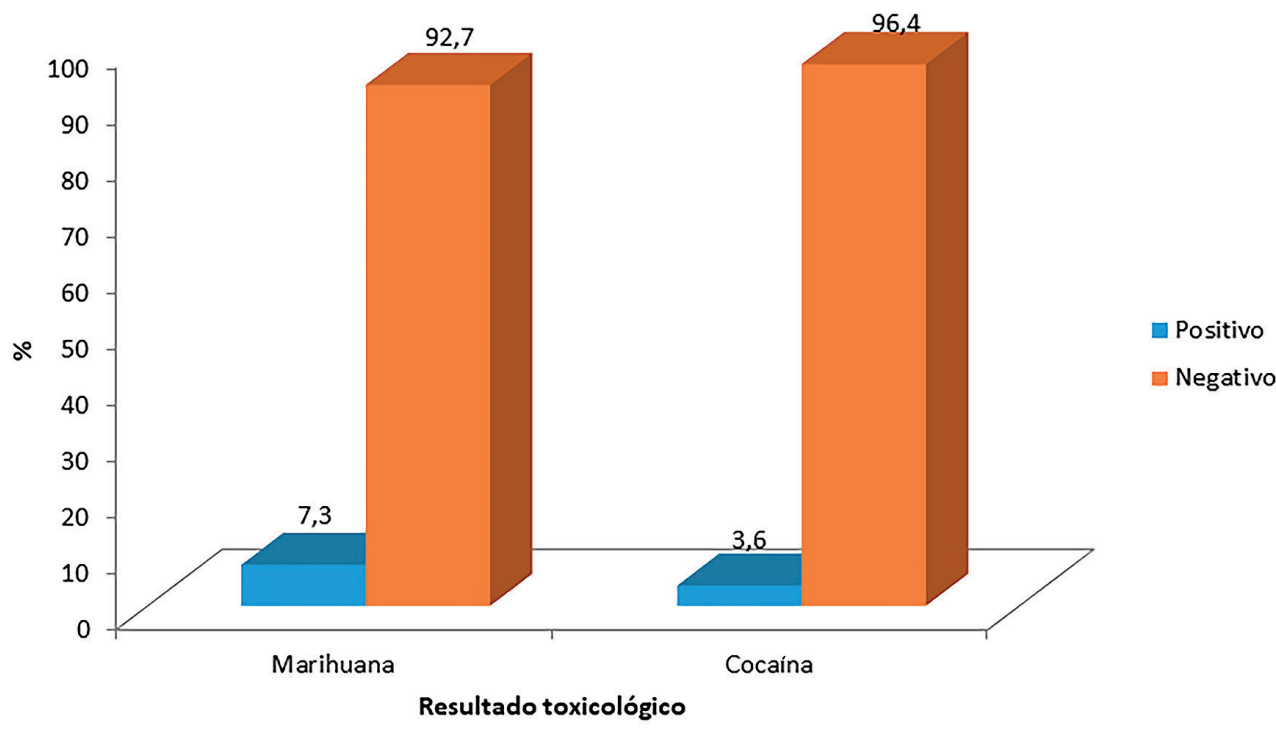

Figura 1. Resultados toxicológicos obtenidos en la población de estudio 


\section{Características asociadas al consumo de marihuana y cocaína}

Se encontró una asociación (diferencias) estadísticamente significativa entre el resultado positivo de marihuana y las variables sexo, edad, nivel de escolaridad y tipo de empresa. Al realizar este mismo análisis para cocaína, se encontró asociación con las mismas variables, excepto con edad (tablas 2 y 3 ).
En las personas que optaban por un empleo en empresas del sector de la construcción, se encontró que aproximadamente 16 de cada 100 sujetos presentaron consumo de marihuana y en el sector de la manufactura 9 de cada 100 consumían esta misma sustancia. En cuanto al consumo de cocaína, la mayor prevalencia se observó en el sector agrícola y en el de la construcción, con 10,7\% y 10,2\% respectivamente (tablas 2 y 3 ).

Tabla 2. Características de la población de estudio y su asociación con el resultado toxicológico para marihuana

\begin{tabular}{|c|c|c|c|c|c|}
\hline \multirow{3}{*}{ Características } & \multicolumn{4}{|c|}{ Marihuana (resultado) } & \multirow[b]{3}{*}{ Valor $\mathrm{p}$} \\
\hline & \multicolumn{2}{|c|}{ Negativo } & \multicolumn{2}{|c|}{ Positivo } & \\
\hline & Frecuencia & $\%$ & Frecuencia & $\%$ & \\
\hline
\end{tabular}

Sexo

$\begin{array}{lccccc}\text { Femenino } & 236 & 98,3 \% & 4 & 1,7 \% & \\ \text { Masculino } & 1.762 & 92,0 \% & 154 & 8,0 \% & 0,000^{*}\end{array}$

Grupos de

\begin{tabular}{llccccc} 
edad & $14-25$ & 815 & $90,7 \%$ & 84 & $9,3 \%$ & \\
& $26-35$ & 778 & $92,0 \%$ & 68 & $8,0 \%$ & \\
& 36 y más & 405 & $98,5 \%$ & 6 & $1,5 \%$ & $0,000^{*}$ \\
\multirow{5}{*}{ Escolaridad } & Bachillerato & 900 & $91,1 \%$ & 88 & $8,9 \%$ & \\
& Primaria & 69 & $89,6 \%$ & 8 & $10,4 \%$ & \\
& Sin datosional & 149 & $96,8 \%$ & 5 & $3,2 \%$ & \\
& Técnico & 553 & $92,8 \%$ & 43 & $7,2 \%$ & \\
& Tecnólogo & 197 & $95,2 \%$ & 10 & $4,8 \%$ & \\
\multirow{2}{*}{ Tipo de } & 130 & $97,0 \%$ & 4 & $3,0 \%$ & $0,015^{*}$ \\
empresa & Transporte & 696 & $96,1 \%$ & 28 & $3,9 \%$ & \\
& Manufactura & 1.089 & $91,2 \%$ & 105 & $8,8 \%$ & \\
& Vig. privada & 112 & $91,8 \%$ & 10 & $8,2 \%$ & \\
& Agrícola & 27 & $96,4 \%$ & 1 & $3,6 \%$ & \multirow{2}{*}{$0,000^{*}$} \\
\hline
\end{tabular}

* Asociación estadística según prueba chi cuadrado de Pearson. Valor $p<0,05$.

Fuente: elaboración propia. 
Tabla 3. Características de la población de estudio y su asociación con el resultado toxicológico para cocaína

\begin{tabular}{|c|c|c|c|c|c|}
\hline \multirow{3}{*}{ Características } & \multicolumn{4}{|c|}{ Cocaína (resultado) } & \multirow[b]{3}{*}{ Valor $p$} \\
\hline & \multicolumn{2}{|c|}{ Negativo } & \multicolumn{2}{|c|}{ Positivo } & \\
\hline & Frecuencia & $\%$ & Frecuencia & $\%$ & \\
\hline
\end{tabular}

Sexo

$\begin{array}{lccccc}\text { Femenino } & 240 & 100,0 \% & 0 & 0,0 \% & \\ \text { Masculino } & 1.878 & 96,0 \% & 79 & 4,0 \% & 0,002^{*}\end{array}$

\begin{tabular}{|c|c|c|c|c|c|c|}
\hline \multicolumn{7}{|l|}{ Grupos de } \\
\hline edad & $14-25$ & 877 & $96,2 \%$ & 35 & $3,8 \%$ & \\
\hline & $26-35$ & 831 & $96,0 \%$ & 35 & $4,0 \%$ & \\
\hline & 36 y más & 410 & $97,9 \%$ & 9 & $2,1 \%$ & $0,204^{*}$ \\
\hline \multirow{6}{*}{ Escolaridad } & Bachillerato & 958 & $95,3 \%$ & 47 & $4,7 \%$ & \\
\hline & Primaria & 75 & $96,2 \%$ & 3 & $3,8 \%$ & \\
\hline & Profesional & 157 & $98,7 \%$ & 2 & $1,3 \%$ & \\
\hline & Sin datos & 585 & $96,1 \%$ & 24 & $3,9 \%$ & \\
\hline & Técnico & 209 & $98,6 \%$ & 3 & $1,4 \%$ & \\
\hline & Tecnólogo & 134 & $100,0 \%$ & 0 & $0,0 \%$ & $0,015^{*}$ \\
\hline \multirow{5}{*}{$\begin{array}{l}\text { Tipo de } \\
\text { empresa }\end{array}$} & Transporte & 715 & $98,8 \%$ & 9 & $1,2 \%$ & \\
\hline & Manufactura & 1.180 & $95,5 \%$ & 55 & $4,5 \%$ & \\
\hline & Vig. privada & 119 & $97,5 \%$ & 3 & $2,5 \%$ & \\
\hline & Agrícola & 25 & $89,3 \%$ & 3 & $10,7 \%$ & \\
\hline & Construcción & 79 & $89,8 \%$ & 9 & $10,2 \%$ & $0,000^{*}$ \\
\hline
\end{tabular}

* Asociación estadística según prueba chi cuadrado de Pearson. Valor p <0,05.

Fuente: elaboración propia.

\section{Discusión}

El consumo de alcohol y de otras drogas presenta una elevada prevalencia en la sociedad en general y también entre la población trabajadora, lo que repercute sobre el medio laboral. Los efectos del consumo de estas sustancias en el medio laboral son muy importantes por las implicaciones que tienen para el trabajador consumidor, sus compañeros y la empresa (enfermedades, accidentes labora- les, ausentismo, incapacidades laborales, disminución de la productividad, entre otros) (10).

En Estados Unidos, para prevenir los consumos en los lugares de trabajo, asumen que este puede prevenirse contratando solo a las personas que no consumen drogas cuando solicitan un puesto vacante. Existe evidencia de que los exámenes toxicológicos complementarios preocupacionales, para 
dar cuenta del consumo de drogas, son aceptados en procesos de búsqueda de empleo $(10,11)$.

Con respecto a la predicción del consumo de drogas en ambientes laborales, la gran mayoría de la investigaciones se han centrado en los factores estresantes del trabajo (por ejemplo, demandas excesivas, acoso laboral, estrés laboral, rutinas extremas, altas temperaturas, etc.) como posibles causas del consumo de sustancias por parte de los empleados $(12,13)$. No hay estudios que predigan si un consumidor de drogas que aspira a un trabajo puede continuar consumiendo, pero sí existe evidencia que si bien algunos consumos pueden resultar no problemáticos, otros pueden avanzar y convertirse en desadaptativos (14-16), lo que sí implica serios problemas cuando de aspirar a un vacante de un trabajo de riesgo se trata. Otra de las teorías en las que se basa la hipótesis, es que las personas más propensas a abusar del alcohol y otras sustancias pueden tener otras conductas que aumentan el riesgo de lesión, un concepto denominado propensión a la desviación (17).

El concepto de propensión a la desviación plantea la hipótesis de que, entre los trabajadores, el consumo excesivo de sustancias o el uso indebido es una constelación de conductas desviadas que también pueden incluir una mayor asunción de riesgos, búsqueda de sensaciones e incumplimiento con políticas de seguridad en el lugar de trabajo (18).

En otras palabras, el uso drogas puede 262 ser un síntoma de una estructura psíquica subyacente que aumenta el riesgo de los individuos de lesionarse en el trabajo, estando relacionada la lesión con el consumo de la sustancia. De hecho, la investigación empírica que ha tenido en cuenta otros factores de riesgo: la impulsividad, la baja percepción del riesgo y los trastornos mentales asociados, el estilo atribucional ha encontrado que la asociación entre el uso de sustancias psicoactivas y las lesiones, a menudo se atenúan al controlar esos otros factores, lo que respalda esta teoría (18).

En cuanto a las repercusiones del consumo de alcohol y otras drogas en el medio laboral, según estudios de la ILO (19), los trabajadores que las consumen presentan un ausentismo laboral de dos a tres veces mayor que el resto de los trabajadores. Las bajas por enfermedad tienen un costo tres veces mayor a las del resto de los empleados, a la vez que esas mismas personas perciben también indemnizaciones por un importe cinco veces mayor que el resto de sus compañeros. Refiere también que el 20-25\% de los accidentes laborales ocurren en trabajadores que se encuentran bajo los efectos del alcohol u otras drogas, $y$ causan lesiones a ellos mismos o a otros. Además, el consumo de drogas afecta negativamente la productividad, lo que ocasiona importantes perjuicios a empresarios y trabajadores por desajustes laborales, rotación del personal, reducción del rendimiento laboral y mala calidad del trabajo (20).

De otro lado, algunos autores han encontrado que existe cierta evidencia de que las tasas de ausentismo (6) y la rotación del personal (21) son más altas entre los usuarios de drogas. Del mismo 
modo, algunos estudios sugieren que los usuarios de drogas pueden ser más propensos a estar involucrados en accidentes (22), aunque esta asociación puede estar limitada a hombres jóvenes (23).

Con respecto a los hallazgos encontrados en este estudio, relacionados con las drogas más consumidas y su asociación con edad, sexo y empresa para la cual aspira el candidato, hay evidencias encontradas por otros autores que han indicado que la asociación entre el uso de sustancias con el trabajo está moderado por variables como el sexo (24), la edad (25) y la industria (26), algo muy similar a lo encontrado en el presente estudio.

En relación con las sustancias encontradas en las pruebas de drogas en este trabajo, que fueron la marihuana y la cocaína, los hallazgos de estudios experimentales han encontrado deficiencias en el tiempo de reacción, razonamiento, coordinación, cuidado y juicio que pueden ser explicados incluso por cantidades mínimas de uso de sustancias, lo que puede aumentar el riesgo de un trabajador de lesionarse en el lugar de trabajo (27).

El uso de cannabis afecta negativamente la capacidad de realizar tareas complejas que requieren de atención y concentración, sumado a la disminución de los reflejos y al aumento del riesgo de accidentes laborales (28). Muchos consumidores de cocaína pueden tener problemas, tanto en tareas que requieran atención y concentración, como en la abrupta toma de decisiones sin análisis previos de los riesgos y consecuencias (29).
Debido a la coyuntura actual de la normativa colombiana en relación con la legalización de la marihuana para usos medicinales, se hace mención especial a esta por referirse a las implicaciones que esto tendrá en los lugares de trabajo. Empleadores de Canadá ya han mostrado su preocupación y se han referido al tema, afirmando que la marihuana ya es la sustancia más comúnmente encontrada en las pruebas de drogas en el lugar de trabajo (30) y esperan que su uso crezca exponencialmente tras el uso medicinal luego con la legalización completa (31). Existe una preocupación generalizada entre los empleadores de que el aumento en el consumo de cannabis, liderado por la normalización social, dará lugar a una mayor incidencia de deterioro en el lugar de trabajo. Muchos empleadores esperan que aumenten los accidentes en el lugar de trabajo, especialmente en industrias sensibles a la seguridad y, por tanto, se están preparando para crear políticas que cubran adecuadamente los problemas en el trabajo que puedan surgir con la legalización de la marihuana medicinal o recreativa.

El primer enfoque es una política de "tolerancia cero", según la cual ningún nivel de consumo de cannabis se considera seguro antes de conducir o en trabajos de alto riesgo (32). El segundo enfoque es establecer un "límite per se" sobre los niveles aceptables de cannabis en la sangre, similar a las pautas del contenido de alcohol en sangre. Existen ventajas para ambos enfoques, pero ninguno proporciona una definición estándar para lo que constituye impedimento y sobre todo en la conducción y los lugares de trabajo con oficios de alto riesgo. Se su- 
giere que, como mínimo, debe haber una prohibición expresa sobre el uso de marihuana en lugares de trabajo sensibles a la seguridad (31).

Finalmente y en relación con las empresas involucradas en este estudio: transporte, manufactura, vigilancia privada, agrícolas y construcción, es necesario anotar que todas en general tienen entre sus puestos de trabajo labores de alto riesgo $\mathrm{y}$, por tanto, incluyen entre los exámenes complementarios preocupacionales pruebas toxicológicas de drogas en orina, lo cual coincide con lo sugerido por otros estudios (26).

\section{Conclusiones}

El uso de pruebas de drogas en orina, como exámenes complementarios para las valoraciones medicas preocupacionales, es el primer paso para la prevención del consumo de drogas en los ambientes laborales de alto riesgo, dado que hay evidencia sobre la probabilidad de que los aspirantes a un trabajo que participan en comportamientos dañinos y que consumen sustancias tienden a asumir mayores riesgos en el trabajo.

Esto no significa que la relación entre el uso de sustancias y las lesiones labora- les sea espuria, sino que las políticas y las estrategias de intervención centradas en el uso de sustancias en los lugares de trabajo deben abordar las características y los rasgos subyacentes de las personas con determinados perfiles de riesgo.

\section{Limitaciones}

Los resultados no fueron confirmados mediante métodos cromatográficos (cromatografía de gases y líquidos con detección por espectrometría de masas), lo cual puede dejar la duda de falsos positivos o negativos, que en la actualidad pueden ser frecuentes gracias a la interacción con medicamentos o a los fraudes a los que acuden las personas para evitar ser detectados. Aunque muchos de estos aspectos fueron controlados, el estándar de oro lo constituyen las pruebas confirmatorias.

\section{Conflicto de intereses}

Los autores declaran no tener conflicto de intereses.

\section{Financiación}

El estudio no requirió financiación externa. 


\section{Referencias bibliográficas}

1. World Health Organization. Regional guidelines for the development of healthy workplaces [Internet]. 1999 [citado 2018 my. 7]. Disponible en: http://www.who. int/occupational_health/publications/ wproguidelines/en/

2. Organización Internacional del Trabajo, OIT. Iniciativas en el lugar de trabajo para prevenir y reducir los problemas causados por el alcohol y otras drogas. Ginebra: OIT; 1988.

3. Organización Internacional del Trabajo, OIT. Proyecto de repertorio de recomendaciones prácticas sobre el tratamiento en los lugares de trabajo de los problemas que plantea el consumo de alcohol y drogas. Ginebra: OIT; 1994.

4. Organización Internacional del trabajo, OIT. Lucha contra el abuso de alcohol y drogas en el lugar de trabajo. Ginebra: OIT; 1998.

5. Health and safety executive. Homeworkers: guidance for employers on health and safety [Internet]. 2011 [citado 2018 feb 24]. Disponible en: http://www. hse.gov.uk/pubns/indg226.pdf

6. Normand J, Salvards S, Mahoney J. An evaluation of pre-employment drug testing. J Appl Psychol. 1990;75(6):629-39.

7. Ministerio de Salud y Protección Social. Decreto 2090 [Internet]. 2003 [citado 2018 feb 24]. Disponible en: https://docs. supersalud.gov.co/PortalWeb/Juridica/ Decretos/D2090003.pdf

8. Presidencia de la República. Decreto 1108 [Internet]. 1994 [citado 2018 mar 15]. Disponible en: http:// www.descentralizadrogas.gov.co/ project/decreto-1108-de-1994-disposiciones-en-relacion-con-el-porte-y-consumo-de-estupefacientes-y-sustancias-psicotropicas/
9. Pontificia Universidad Javeriana. Encuesta Nacional de Salud Mental Colombia [Internet]. 2015 [citado 2018 feb 20]. Disponible en: https://www.javerianacali. edu.co/sites/ujc/files/node/field-documents/field_document_file/saludmental_ final_tomoi_color.pdf

10. Zhang, Z., Huang, L. X., \& Brittingham, A. (1999). Worker drug use and workplace policies and programs: results from the 1994 and 1997 National household survey on drug abuse. Rockville, MD: Dept. of Health and Human Services, Substance Abuse and Mental Health Services Administration.

11. Mastrangelo P. Do college students still prefer companies without employment drug testing? J Bus Psychol. 1997;11:325-37.

12. Frone M. Work stress and alcohol use. Alcohol Res Health. 1999;23:284-91.

13. Frone MR. Are work stressors related to employee substance use? The importance of temporal context assessments of alcohol and illicit drug use. J Appl Psychol. 2008 en.;93(1):199-206.

14. Chassin L, Presson CC, Pitts SC, Sherman SJ. The natural history of cigarette smoking from adolescence to adulthood in a midwestern community sample: multiple trajectories and their psychosocial correlates. Health Psychol. 2000 my.;19(3):223-31.

15. Flory K, Lynam D, Milich R, Leukefeld C, Clayton R. Early adolescent through young adult alcohol and marijuana use trajectories: early predictors, young adult outcomes, and predictive utility. Dev Psychopathol. 2004;16(1):193-213.

16. Oliva A, Parra A, Sánchez I. Consumo de sustancias durante la adolescencia: trayectorias evolutivas y consecuencias para el ajuste psicológico. Int J Clin Health Psychol. 2008;8:153-69. 
17. Dawson DA. Heavy drinking and the risk of occupational injury. Accid Anal Prev. 1994 oct.;26(5):655-65.

18. Spicer RS, Miller TR, Smith GS. Worker substance use, workplace problems and the risk of occupational injury: a matched case-control study. J Stud Alcohol [Internet]. 2003 jul. [citado 2018 my. 7];64(4):570-8. Disponible en: http://www. jsad.com/doi/10.15288/jsa.2003.64.570

19. International Labour Organization. Occupational Safety and Health [Internet]. Advancing social justice, promoting decent work. 2006 [citado 2018 my. 7]. Disponible en: http://www.ilo.org/safework/lang--en/index.htm

20. Howland J, Almeida A, Rohsenow D, Minsky S, Greece J. How safe are federal regulations on occupational alcohol use? J Public Health Policy [Internet]. 2006 [citado 2018 my. 7];27(4):389404. Disponible en: http://www.jstor.org/ stable/4125181

21. Normand J, Salyards S. An empirical evaluation of preemployment drug testing in the United States Postal Service: interim report of findings. NIDA Res Monogr. 1989;91:111-38.

22. Hingson RW, Lederman RI, Walsh DC. Employee drinking patterns and accidental injury: a study of four New England states. J Stud Alcohol. 1985 jul.;46(4):298-303.

23. Kaestner R, Grossman M. The effect of drug use on workplace accidents. Labour Econ [Internet]. 1998 sept. [citado 2018 my. 7];5(3):267-94. Disponible en: http://linkinghub.elsevier.com/retrieve/pii/ S0927537197000213

24. Kaestner R, Grossman M. Wages, workers' compensation benefits, and drug use: indirect evidence of the effect of drugs on workplace accidents. Am Econ Rev [Internet]. 1995;85(2):5560. Disponible en: http://www.jstor.org/ stable/2117891
25. Pollack ES, Franklin GM, Fulton-Kehoe D, Chowdhury R. Risk of job-related injury among construction laborers with a diagnosis of substance abuse. J Occup Environ Med. 1998 jun.;40(6):573-7.

26. Wickizer TM, Kopjar B, Franklin G, Joesch J. Do drug-free workplace programs prevent occupational injuries? Evidence from Washington State. Health Serv Res. 2004 febr.;39(1):91-110.

27. National Research Council. Under the Influence? Drugs and the American Work Force [Internet]. Washington, D.C.: National Academies Press; 1994 [citado 2018 my. 7]. Disponible en: http://www. nap.edu/catalog/2118

28. Commission for occupational, safety and health MIAC. Guidance note. Alcohol and other drugs at the workplace [Internet]. 2008 [citado 2018 mar 23]. Disponible en: https://www.commerce.wa.gov. au/sites/default/files/atoms/files/alcohol_ and_drugs_0.pdf

29. Navarro J, Megías E. La incidencia de las drogas en el medio laboral de la Comunidad Valenciana. Fundación de Ayuda contra la Drogadicción y Generalitat Valenciana. Madrid. 1999.

30. Els C, Amin A, Straube S. Marijuana and the Workplace. Can J Addict. 2017;7(4).

31. Enform. Enform's Submission to the Task Force on Marijuana Legalization, Regulation and Restriction [Internet]. 2017 [citado 2018 marzo 17]. Disponible en: http://www.enform.ca/files/EnformLtr-Marijuana_legalization_commission. pdf

32. AAA Foundation. An evaluation of data from drivers arrested for driving under the influence in relation to per se limits for cannabis [Internet]. 2016 [citado 2018 my. 7]. Disponible en: https:// aaafoundation.org/evaluation-data-drivers-arrested-driving-influence-relation-per-se-limits-cannabis/ 